\title{
Quarkonia measurements with STAR
}

\author{
Zhangbu Xu, Thomas Ullrich ${ }^{\mathrm{a}}$ \\ for the STAR Collaboration ${ }^{\mathrm{b}}$ \\ Physics Department, Brookhaven National Laboratory, Upton, NY 11973, USA
}

Received: 12 September 2008 / Revised: 4 March 2009 / Published online: 27 March 2009

(C) Springer-Verlag / Società Italiana di Fisica 2009

\begin{abstract}
We report results on quarkonium production from the STAR experiment at the Relativistic Heavy-Ion Collider (RHIC). $J / \psi$ spectra in $p+p$ and $\mathrm{Cu}+\mathrm{Cu}$ collisions at $\sqrt{s_{N N}}=200 \mathrm{GeV}$ with transverse momenta in the range of $0.5-14 \mathrm{GeV} / c$ and $5-8 \mathrm{GeV} / c$, respectively, are presented. We find that for $p_{T}>5 \mathrm{GeV} / c$ yields in $p+p$ collisions are consistent with those in minimum-bias $\mathrm{Cu}+\mathrm{Cu}$ collisions scaled with the respective number of binary nucleonnucleon collisions. In this range the nuclear modification factor, $R_{A A}$, is measured to be $0.9 \pm 0.2$ (stat). For the first time at RHIC, high- $p_{T} J / \psi$-hadron correlations were studied in $p+p$ collisions. Implications from our measurements on $J / \psi$ production mechanisms, constraints on open bottom yields, and $J / \psi$ dissociation mechanisms at high- $p_{T}$ are discussed. In addition, we give a brief status of measurements of $\Upsilon$ production in $p+p$ and $\mathrm{Au}+\mathrm{Au}$ collisions and present projections of future quarkonia measurements based on an upgrades to the STAR detector and increased luminosity achieved through stochastic cooling of RHIC.
\end{abstract}

PACS 25.75.Cj $\cdot 25.75 . \mathrm{Nq} \cdot 12.38 . \mathrm{Mh} \cdot 14.40 . \mathrm{Gx}$

The dissociation of quarkonia due to color-screening of their constituent quarks in a Quark-Gluon Plasma (QGP) is a classic signature for deconfinement in relativistic heavyion collisions $[1,2]$. The suppression of the various $J / \psi$ and $\Upsilon$ states is determined by their binding energy and the temperature in the plasma. Results from the PHENIX experiment at RHIC show that the suppression of $J / \psi$ as a function of centrality (the number of participants) is similar to that observed by NA50 and NA60 at the CERN-SPS, even though the temperature and energy density reached in these collisions is significantly lower than

\footnotetext{
a e-mail: thomas.ullrich@bnl.gov
}

${ }^{\mathrm{b}}$ url:http://www.star.bnl.gov at RHIC [3-5]. This indicates that at RHIC energies additional mechanisms countering the suppression, such as recombination of charm quarks in the later stage of the collision, may play an important role; they will need to be studied systematically before conclusions from the observed suppression pattern can be drawn. In most theoretical models, the dissociation of quarkonia states is computed at rest relative to the QGP [1,2]. Recently, techniques based on the AdS/CFT duality have been utilized to study the dissociation of quark-antiquark pairs with large velocities relative to the strongly coupled QGP [6]. Calculations in this framework show that bound states of heavy fermion pairs (an analog of quarkonium in QCD) have an effective dissociation temperature that decreases with $p_{T}$, implying an increasing suppression, i.e. decreasing $R_{A A}$, with $p_{T}$. To test this conjecture measurements of $J / \psi R_{A A}$ above $p_{T}>5 \mathrm{GeV} / c$ are needed where the effective $J / \psi$ dissociation temperature is expected to be lower than the temperatures reached in RHIC collisions $\left(\sim 2 T_{c}\right)$ [7-11].

Understanding $J / \psi$ production requires knowing what fraction of $J / \psi \mathrm{s}$ are produced from (i) gluon and heavyquark fragmentation, from (ii) decay feed-down of B mesons and $\chi_{c}$ states, and (iii) what fraction originates from direct production either through a color-octet or color-singlet state. Our understanding of $J / \psi$ production mechanisms has gone through several cycles in recent decades [12-15]. There is no convincing model explaining all the major features of the existing data in $e^{+} e^{-}$annihilation and hadron-hadron collisions. The color-singlet model (CSM) [12-14] underpredicts the $J / \psi$ spectra by an order of magnitude in $\bar{p}+p$ collisions at $\sqrt{s}=1.8 \mathrm{TeV}[12,13]$. The color octet $(\mathrm{COM})$ and color evaporation model (CEM) were proposed to explain the production yields, but fail to explain the recent measurements of large spin alignment (polarization) from the same experiment $[3,4,12,13]$. In addition, $J / \psi$ is found to be dominantly associated with open-charm pair production in $e^{+} e^{-}$annihilation at $\sqrt{s}=10.6 \mathrm{GeV}$ [15]. These later findings suggest that yields calculated from leadingorder pQCD may not be the dominant contribution to the 
$J / \psi$ production. UA1 has analyzed the $J / \psi$-hadron correlations in $\bar{p}+p$ collisions to separate $J / \psi$ sources at high $p_{T}$ from $\chi_{c}$ decay without a near-side correlation and from $B$ hadron decay with a strong near-side correlation $[16,17]$.

In this paper we report on the measurement of $J / \psi$ production at midrapidity with the STAR experiment in $p+p$ and $\mathrm{Cu}+\mathrm{Cu}$ collisions at $\sqrt{s_{N N}}=200 \mathrm{GeV}$ with transverse momenta in the range of $0.5-14 \mathrm{GeV} / c$ and $5-8 \mathrm{GeV} / c$, respectively. In addition, $J / \psi$-hadron correlations, originally proposed and studied by UA1 $[16,17]$ are presented. The technique used is analog to that deployed in hadron-hadron correlations studies in STAR [18]. We will also briefly report on the status of measurements of the $\Upsilon$ in $p+p$ and $\mathrm{Au}+\mathrm{Au}$ collisions and present projections of yields that will become available after completion of the STAR detector upgrades and the increase of RHIC luminosity that will be achieved once stochastic cooling for ion beams is implemented.

In the analyses reported here, the $J / \psi$ and $\Upsilon$ are reconstructed through their decays into electron pairs, $Q \bar{Q} \rightarrow$ $e^{+} e^{-}$. The large acceptance of the tracking system in the STAR Time Projection Chamber (TPC) [19-21] and the electron trigger capability from the Barrel Electromagnetic Calorimeter (BEMC) [19-21] with $|\eta|<1$ are very well suited for such analyses. At STAR, both the TPC and BEMC can provide electron identification [19-21]. At high $p_{T}$, the BEMC is a very powerful tool for electron identification and can be used for online triggering to enrich the electron sample. At moderate $p_{T}$, the TPC provides sufficient energyloss $(d E / d x)$ resolution to identify electrons efficiently.

Various online trigger schemes were deployed to maximize the recorded yield. The low- $p_{T} J / \psi$ trigger is based on two spatially separated BEMC towers with $E_{T}>$ $1.2 \mathrm{GeV}$. A higher level trigger makes a final selection based on the approximated invariant mass of the pair. However, this trigger can only be efficiently used in $p+p$ collisions; in $\mathrm{A}+\mathrm{A}$ collisions this trigger does not provide sufficient discrimination power due to the high hit occupancy in the BEMC. The high- $p_{T} J / \psi$ trigger requires only one tower above a given high-energy threshold. For the data presented here the thresholds were: $E_{T}>3.5 \mathrm{GeV}(p+p$ run 5), $E_{T}>3.75 \mathrm{GeV}\left(\mathrm{Cu}+\mathrm{Cu}\right.$ run 5), and $E_{T}>5.4 \mathrm{GeV}$ (pp run 6), respectively. The $\Upsilon$ trigger is based on a single hightower signal $\left(E_{T}>4.5 \mathrm{GeV}\right)$ and a subsequent pair selection with invariant mass cut performed in an online highlevel trigger system using the full BEMC tower data.

For the $\Upsilon$ measurement the sampled luminosities were $9 \mathrm{pb}^{-1}$ in $p+p$ collisions and $12 \mathrm{pb}^{-1}(p+p$ equivalent) in $\mathrm{Au}+\mathrm{Au}$ collisions. The low- $p_{T} J / \psi$ is from a dataset with $0.4 \mathrm{pb}^{-1}$ of $p+p$ luminosity. The data used for the high- $p_{T} J / \psi$ analysis was recorded during the $p+p$ and $\mathrm{Cu}+\mathrm{Cu}$ runs in 2005 and the $p+p$ run in 2006 using the trigger setup described above in coincidence with a minimum bias trigger. The minimum bias trigger is based on the coincidence of two beam-beam counter (BBC) in $p+p$ and on the coincidence between two Zero Degree Calorimeters (ZDCs) in $\mathrm{Cu}+\mathrm{Cu}$. The integrated luminosity was $\sim 2.8(11.3) \mathrm{pb}^{-1}$ for $p+p$ collisions collected in year 2005 (2006), and $\sim 860 \mu \mathrm{b}^{-1}\left(3 \mathrm{pb}^{-1} p+p\right.$ equivalent) for $\mathrm{Cu}+\mathrm{Cu}$ collisions. In $\mathrm{Cu}+\mathrm{Cu}$ data, the most central $0-60 \%$ of the total hadronic cross-section was selected by using the uncorrected charged particle multiplicity at midrapidity $(|\eta|<0.5)$ [7-10, 19-21].

For the high- $p_{T} J / \psi$ analysis, we required one high- $p_{T}$ electron identified with the combination of BEMC tower energy, shower shape (from shower-max detectors embedded in the BEMC), and $d E / d x$ measured in the TPC. The $p_{T}$ cut for the second electron was substantially lower and only $d E / d x$ in the TPC was used for identification.

Figure 1 shows the invariant mass spectra for the high$p_{T}$ dielectron sample in $p+p$ collisions from runs 5 and 6 (2005 and 2006) and in $\mathrm{Cu}+\mathrm{Cu}$ collisions (run 5). The combinatorial background, derived from like-sign pairs, is depicted by dashed lines. The $J / \psi$ signal is extracted in the mass window $2.9<M_{\text {inv }}<3.2 \mathrm{GeV} / c^{2}$. Due to the high- $p_{T}$ cuts used in this study the signal-to-background ratio $(\mathrm{S} / \mathrm{B})$ is exceptionally large. In $p+p$ collisions we obtain $S / B=$ $22 / 2(40 / 14)$ for run 5 (6) and $17 / 23$ in $\mathrm{Cu}+\mathrm{Cu}$ collisions. The $p_{T}$ coverage in $p+p$ and $\mathrm{Cu}+\mathrm{Cu}$ collisions taken in

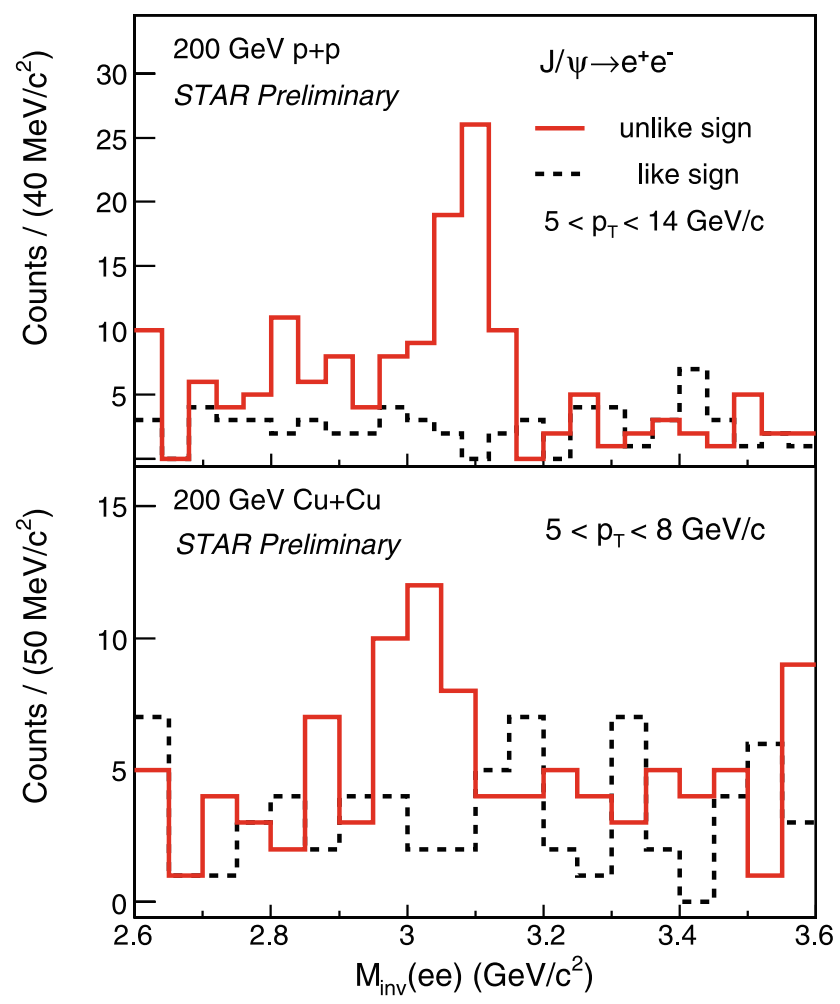

Fig. 1 The $e^{+} e^{-}$invariant mass distribution in $p+p$ collisions and $\mathrm{Cu}+\mathrm{Cu}$ collisions at $\sqrt{s_{N N}}=200 \mathrm{GeV}$. The solid and dashed lines represent the distribution of un-like and like-sign pairs, respectively 
run 5 is $5<p_{T}<8 \mathrm{GeV} / c$. For $p+p$ collisions in run 6 the larger integrated luminosity, the larger BEMC coverage, and optimized trigger thresholds allow us to extend the $p_{T}$ reach to $14 \mathrm{GeV} / c$. Figure 2 shows the fully corrected $J / \psi$ invariant cross-section $B_{e e} \times E d^{3} \sigma / d^{3} p$ as a function of $p_{T}$ in $p+p$ collisions for all three datasets. Our spectrum is in good agreement with results from the PHENIX experiment, which is plotted for comparison [22].

Figure 3 shows the $J / \psi$ nuclear modification factors $R_{A A}$ as a function of $p_{T}$ in $0-20 \%$ and $0-60 \% \mathrm{Cu}+\mathrm{Cu}$ collisions calculated from PHENIX [23, 24] and STAR mea-

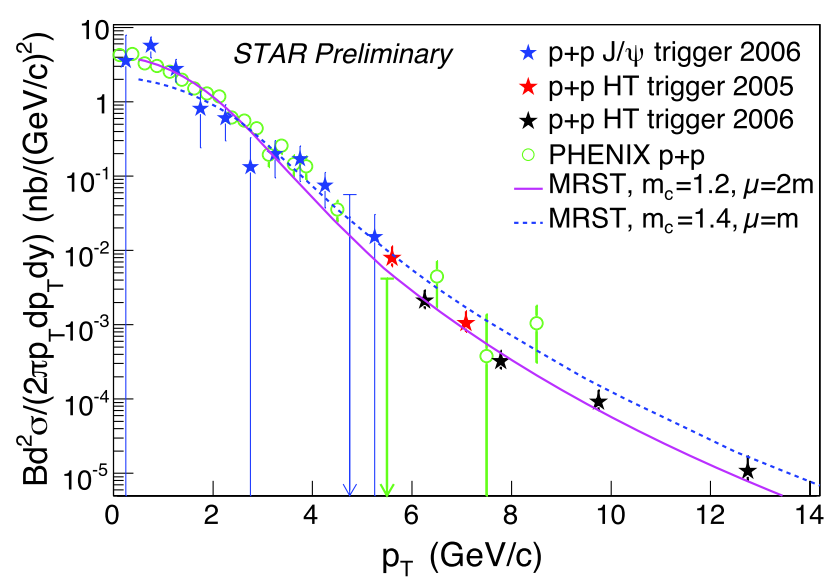

Fig. $2 J / \psi$ invariant cross-section times the di-electron branching ratio as a function of $p_{T}$ in $p+p$ collisions at $\sqrt{s}=200 \mathrm{GeV}$. The lines correspond to pQCD with Color Evaporation Model (CEM) calculations $[3,4]$

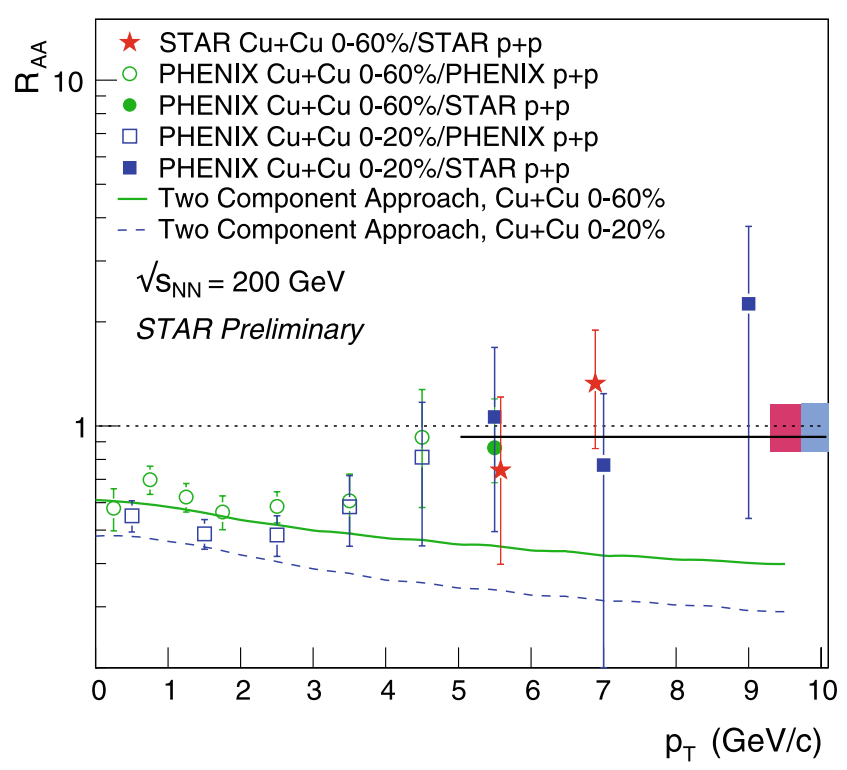

Fig. $3 J / \psi R_{A A}$ as a function of $p_{T}$. The solid line represents the fit to all the data points at $5<p_{T}<10 \mathrm{GeV} / c$ yielding $R_{A A}=0.9 \pm 0.2$ (stat). The curves are "Two Component Approach" model prediction [25]. The boxes in the right show the normalization uncertainty for $0-60 \%$ (left) and 0-20\% (right) collisions surements. The data suggest that $R_{A A}$ is rising towards unity for $p_{T}>5 \mathrm{GeV} / c$, although the large errors currently preclude strong conclusions. A combined fit to all high $p_{T}$ data points above $5 \mathrm{GeV} / c$ gives $R_{A A}=0.9 \pm 0.2$ (stat), consistent with unity and $2 \sigma$ higher than that at low- $p_{T}\left(R_{A A} \sim\right.$ $0.5)$. The systematic uncertainties are still under evaluation. This result is in contradiction with expectations from AdS/CFT-based [6] and Two-Component-Approach [25] models that predict a decrease in $R_{A A}$ with increasing $p_{T}$. A similar trend was also observed by NA60 Collaboration in In + In collisions at $\sqrt{s_{N N}}=17.3 \mathrm{GeV}$ [26]. There, however, $R_{A A}$ reaches unity at considerably smaller $p_{T}$ than at RHIC, suggesting that the effects are most likely of a different physics origin. Our results could indicate that other $J / \psi$ production mechanisms that counter the suppression such as recombination and formation time effects [27, 28] play an increasingly dominant role at higher $p_{T}$. The small suppression of the $J / \psi$ at high- $p_{T}$ stands in contrast to a substantial suppression of open charm production at similar $p_{T}$ [29-32].

The large $S / B$ ratio of the high- $p_{T} J / \psi$ data in $p+p$ collisions allows us, for the first time at RHIC, to study $J / \psi$ hadron correlations, which can potentially provide important constraints on the underlying $J / \psi$ production mechanisms. Figure 4 shows the azimuthal angle correlations $d N / d \Delta \phi$ per $J / \psi$ between $J / \psi$ s with $p_{T}>5 \mathrm{GeV} / c$ and charged hadrons with $p_{T}>0.5 \mathrm{GeV} / c$. No significant yield for near-side correlations $(\Delta \phi \sim 0)$ is observed. This is in stark contrast to the case of hadron-hadron correlations [18].

Monte-Carlo simulations (PYTHIA 6.3.19) [33] show a strong near-side correlation dominantly due to the feeddown of $J / \psi$ from $B$-meson decays [16, 17], $B \rightarrow$ $J / \psi+X$. Thus, the comparison of the measured near-side yields with PYTHIA simulations allows one to infer the

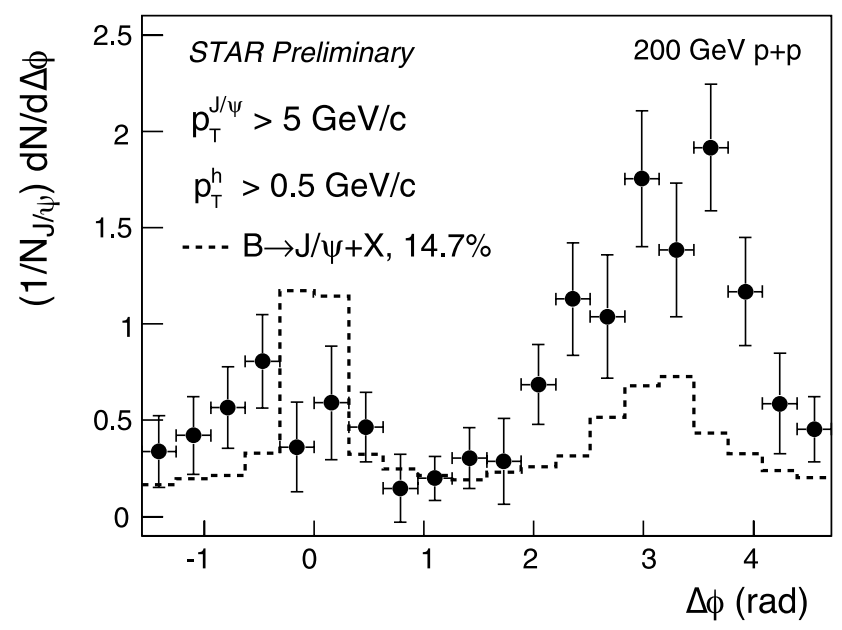

Fig. $4 J / \psi$-hadron azimuthal correlations after background subtraction. The histogram is $J / \psi$-hadron from B decay with full-event simulation from the PYTHIA event generator 


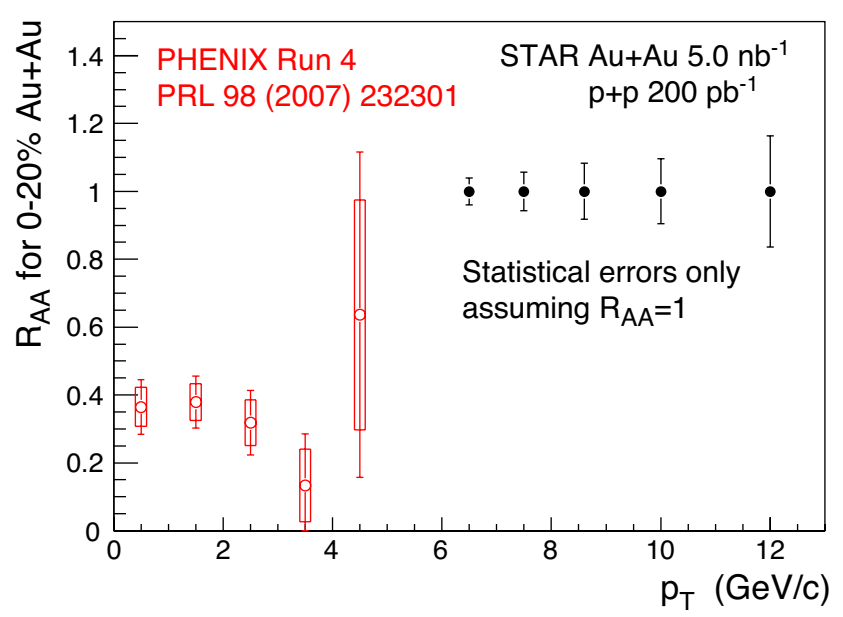

Fig. 5 Projection of $R_{A A}$ at RHIC II luminosity, in which STAR will sample $5 \mathrm{nb}^{-1}$ and $200 \mathrm{pb}^{-1}$ in $\mathrm{Au}+\mathrm{Au}$ and $p+p$ collisions, respectively. The result is compared to $R_{A A}$ at low- $p_{T}$ obtained by PHENIX in run 4 , corresponding to a combination of sampled luminosities of $157 \mu \mathrm{b}^{-1} \mathrm{Au}+\mathrm{Au}$ collisions and $2.6 \mathrm{pb}^{-1} p+p$ collisions

fraction of $J / \psi$ s originating from $B$ meson decays that contribute to the observed yield, albeit in a model dependent way. Assuming no other contribution to the near-side, we obtain an upper limit of $15 \%$ of the $B$-meson feed-down to the inclusive $J / \psi$ cross-section at $p_{T}>5 \mathrm{GeV} / c$. The shape on the near-side is not matched well. A detailed comparison of the spectral shapes is not currently possible due to the limited statistics. This will requires data samples which we hope to collect in the upcoming RHIC run.

It is worth noting that the direct comparison of our inclusive high- $p_{T} J / \psi$ spectra with the feed-down spectra from $\mathrm{pQCD}$ calculations provides less constraints than the method described above. Using NLO predictions for $B$ meson production [34] and decay parameter from measurements by the CLEO collaboration [35] we obtain an upper limit for the $B$ meson feed-down fraction of $\sim 40 \%$ for $p_{T}>5 \mathrm{GeV} / c$. This is in large parts due to mass and scale uncertainties in the NLO calculations [3, 4]. Further studies of $J / \psi$-hadron correlation and $J / \psi$ cross-section with higher statistics will allow us to constrain the $B$ crosssection substantially in the future.

Figure 5 shows a projection of $R_{A A}$ for an integrated luminosity of $5 \mathrm{nb}^{-1}$ for $\mathrm{Au}+\mathrm{Au}$ collisions and $200 \mathrm{pb}^{-1}$ for $p+p$ collisions with an upgraded STAR detector. The key upgrades are: (i) the full barrel time-of-flight detector to improve the electron identification in the low- $p_{T}$ region $\left(p_{T}<3 \mathrm{GeV} / c\right)$ and (ii) the upgrade of the TPC readout electronics in conjunction with an upgrade of the data acquisition system which will increase our data-taking rate tenfold and eliminate dead-time losses for rare triggers. This will allow STAR to make full use of the increased luminosity of RHIC once the stochastic beam cooling is in place.
In addition, with the improved electron identification capabilities and higher data-taking rate the measurement of $J / \psi$ elliptic flow becomes feasible. Simulations show that with the statistics of $10^{9}$ minimum bias $\mathrm{Au}+\mathrm{Au}$ events we will be able to measure $v_{2}$ with $<1 \%$ statistical error. This will provide stringent tests of quark coalescence and charm flow $[3,4]$.

Many of the difficulties that plague the interpretation of the observed charmonium suppression can be avoided when studying bottomonia. Due to the small production crosssection, recombination effects become negligible and absorption by hadronic co-moving matter is unimportant [36]. The small cross-section, however, also makes bottomonium states extremely difficult to measure. While the $\Upsilon(1 S)$ is predicted to be not suppressed at RHIC and even LHC energies, the $\Upsilon(2 S)(\Upsilon(3 S))$ state is assumed to dissociate at temperatures similar to that of the $J / \psi\left(\psi^{\prime}\right)$. The separation of the three states, while possible in STAR, requires substantial statistics which will only become available with the RHIC luminosity upgrades. Detailed $\Upsilon$ simulation and projections can be found in [3, 4].

The STAR experiment already reported on the first RHIC measurement of the $\Upsilon(1 S+2 S+3 S)$ cross-section at midrapidity in $p+p$ collisions at $\sqrt{s}=200 \mathrm{GeV}$. We find $B R \cdot d \sigma /\left.d y\right|_{y=0}=91 \pm 28$ (stat.) \pm 22 (syst.) pb, which is consistent with the world data and NLO pQCD calculations in the CEM [37]. The first ever measurements of $\Upsilon$ mesons in $\mathrm{Au}+\mathrm{Au}$ collisions at $\sqrt{s_{N N}}=200 \mathrm{GeV}$ are underway. We observe a stable signal that will allow us to get first information on the nuclear modification factor of the $\Upsilon$. This will be complemented by measurements in $\mathrm{d}+\mathrm{Au}$ collisions taken in 2008.

In summary, we report measurements of $J / \psi$ spectra from $200 \mathrm{GeV} p+p$ up to transverse momenta of $14 \mathrm{GeV} / c$ and from minimum bias $\mathrm{Cu}+\mathrm{Cu}$ collisions at high $p_{T}$ $\left(5<p_{T}<14 \mathrm{GeV} / c\right)$ at mid-rapidity. The $J / \psi$ nuclear modification factor $R_{A A}$ in $\mathrm{Cu}+\mathrm{Cu}$ at $p_{T}>5 \mathrm{GeV} / c$ is $0.9 \pm 0.2$ (stat) and is about $2 \sigma$ above the values at low $p_{T}$ measured by PHENIX [23, 24]. The study of $J / \psi$-hadron azimuthal correlations show an absence of near-side correlations. Using PYTHIA simulations we derive an upper limit for the fraction of feed-down of $J / \psi$ mesons from $B$ meson decays of $15 \%$ at $p_{T}>5 \mathrm{GeV} / c$. Near future upgrades to the STAR detector and the RHIC machine will allow us to make substantial contributions to the understanding of quarkonia production and provide detailed information of their interaction with the hot dense matter created at RHIC.

Acknowledgements This work has been supported under DOE contract DE-AC02-98CH10886. 


\section{References}

1. T. Matsui, H. Satz, Phys. Lett. B 178, 416 (1986)

2. F. Karsch, D. Kharzeev, H. Satz, Phys. Lett. B 637, 75 (2006). arXiv:hep-ph/0512239

3. A.D. Frawley, T. Ullrich, R. Vogt, Phys. Rept. 462, 125-175 (2008)

4. R. Vogt, Private communications

5. A. Adare et al., Phys. Rev. Lett. 98, 232301 (2007). arXiv:nucl-ex/ 0611020

6. H. Liu, K. Rajagopal, U.A. Wiedemann, Phys. Rev. Lett. 98, 182301 (2007)

7. K. Adcox et al., Nucl. Phys. A 757, 184 (2005). arXiv:nucl-ex/ 0410003

8. B.B. Back et al., Nucl. Phys. A 757, 28 (2005). arXiv:nucl-ex/ 0410022

9. J. Adams et al., Nucl. Phys. A 757, 102 (2005). arXiv:nucl-ex/ 0501009

10. I. Arsene et al., Nucl. Phys. A 757, 1 (2005). arXiv:nucl-ex/ 0410020

11. A. Adare et al., arXiv:nucl-ex/0804.4168

12. F. Abe et al., Phys. Rev. Lett. 69, 3704 (1992)

13. D. Acosta et al., Phys. Rev. D 71, 032001 (2005)

14. M. Kramer, Prog. Part. Nucl. Phys. 47, 141 (2001). arXiv:hep-ph/ 0106120

15. K. Abe et al. (The Belle Collaboration), Phys. Rev. Lett. 89, 142001 (2002). arXiv:hep-ex/0205104

16. C. Albajar et al., Phys. Lett. B 256, 112 (1991)
17. C. Albajar et al., Phys. Lett. B 200, 380 (1988)

18. J. Adams et al., Phys. Rev. Lett. 95, 152301 (2005)

19. M. Anderson et al., Nucl. Instrum. Methods A 499, 659 (2003)

20. M. Beddo et al., Nucl. Instrum. Methods A 499, 725 (2003)

21. B.I. Abelev et al., Phys. Rev. Lett. 98, 192301 (2007)

22. A. Adare et al., Phys. Rev. Lett. 98, 232002 (2007)

23. A. Adare et al., Phys. Rev. Lett. 101, 122301 (2008). arXiv: nucl-ex/0801.0220

24. M.R. Cosentino et al., arXiv:nucl-ex/0806.0353

25. X. Zhao, R. Rapp, arXiv:0712.2407, private communication for $\mathrm{Cu}+\mathrm{Cu}$

26. R. Arnaldi et al., Phys. Rev. Lett. 99, 132302 (2007)

27. F. Karsch, R. Petronzio, Phys. Lett. B 193, 105 (1987)

28. J.P. Blaizot, J.Y. Ollitrault, Phys. Lett. B 199, 499 (1987)

29. B.I. Abelev et al., Phys. Rev. Lett. 98, 192301 (2007)

30. S.S. Adler et al., Phys. Rev. Lett. 96, 032301 (2006)

31. B.I. Abelev et al., arXiv:0805.0364

32. J. Adams et al., Phys. Rev. Lett. 94, 62301 (2005)

33. T. Sjöstrand, Comput. Phys. Commun. 82, 74 (1994)

34. M. Cacciari, P. Nason, R. Vogt, Phys. Rev. Lett. 95, 122001 (2005). arXiv:hep-ph/0502203

35. S. Anderson et al. (The CLEO Collaboration). Phys. Rev. Lett. 89, 282001 (2002)

36. Z.W. Lin, C.M. Ko, Phys. Lett. B 503, 104 (2001). arXiv:nucl-th/ 0007027

37. D. Das et al., arXiv:0804.4504 